\title{
Trials effects in the evoked cardiac response under processing load
}

\author{
ROBERT J. BARRY \\ University of New South Wales, Kensington, N.S.W., Australia
}

\begin{abstract}
Recent studies of the phasic evoked cardiac response in the OR context have identified the deceleratory heart rate component with primary bradycardia. Cardiac deceleration thus appears to reflect stimulus registration, a preliminary process in OR elicitation. Situational factors may lead to an additional acceleratory component, probably reflecting cognitive processing demand. The present study investigated trials effects in these two components of a complex cardiac response, reflecting both stimulus and situational factors. The deceleratory component failed to show a simple trials effect, a finding compatible with the concept of stimulus registration. In contrast, the acceleratory component increased over trials, compatible with its hypothesized reflection of cognitive processing.
\end{abstract}

The study of the phasic cardiac response in man, as elicited by a simple stimulus, has involved an increasing range of perspectives in recent years. An emphasis on the role of situational determinants derives from the work of J. I. Lacey (1967) and his stimulus intake-rejection hypothesis: intake is accompanied by cardiac deactivation, whereas rejection is accompanied by cardiac activation. In contrast, Sokolov's work on the orienting response (OR) has led to many studies of the role of stimulus parameters in phasic response generation, although the classical OR literature made little reference to cardiac activity.

Graham and Clifton (1966) attempted to integrate these perspectives by identifying stimulus intake and heart rate (HR) deceleration with the OR, but such an integration has been questioned on a number of grounds. For example, Velden and Schumacher (1979) commented upon the conceptual mismatch between J. I. Lacey's concept of "situation," which included the subject's intended response as well as the objective aspects of the stimulus, and Graham and Clifton's emphasis on the latter, as exemplified by the restriction of their review to indifferent stimuli. A recent review by Bohlin and Kjellberg (1979), although concentrating upon two-stimulus paradigms, amply illustrates that stimulus and situational factors differentially affect the cardiac response. In a two-stimulus paradigm, such as a warned reaction time task, it is common for the first stimulus to elicit HR deceleration (D1) followed by acceleration (A) and a second deceleration (D2) before the second stimulus. Bohlin and Kjellberg concluded that D1 could be considered an OR to the first stimulus, whereas D2 probably reflected an expectancy process. It appears reasonable to identify $A$ as reflecting

My thanks are extended to Felicia Mitchell for assistance with data collection and analysis. Address reprint requests to Robert J. Barry, School of Education, University of New South Wales, Kensington 2033, Australia. information-processing requirements (Coles \& DuncanJohnson, 1975; Walter \& Porges, 1976). These components were thought by Bohlin and Kjellberg to reflect independent processes, throwing into question the empirical basis of Graham and Clifton's proposed integration.

A corollary of this integration, the conclusion that cardiac deceleration represents an OR, also has been questioned. An important property of the OR, and hence a requirement for purported OR indicators, is habituation with repeated stimulus presentation. A number of studies (e.g., Barry, 1977a, 1977b; Hulstijn, 1978; van Olst, Heemstra, \& ten Kortenaar, 1979; Velden \& Schumacher, 1979) have failed to support this proposition. No study has reported trials effects in HR deceleration comparable with those routinely noted with that exemplar of the OR, GSR. This observation, coupled with the relative insensitivity of $H R$ deceleration to variations in stimulus intensity within the OR context, led to the proposal that HR deceleration reflects an early stage of stimulus processing, a preliminary process in OR elicitation, rather than the OR itself (Barry, 1981, 1982, 1984b). Support for this view has come from the linking of HR deceleration in the OR context to the primary bradycardia of B. C. Lacey and J. I. Lacey $(1977,1978,1980)$.

The Laceys had shown that brief stimuli prolonged the cardiac cycle in which the stimulus onset occurred and that this effect depended upon the relative timing of the stimulus. Early stimulation produces its greatest effect in the concurrent cycle, whereas later stimulation has a smaller effect in that cycle but an increasing effect in the subsequent cardiac cycle. This reflex bradycardia was considered by the Laceys to reflect an "early process of stimulus-registration'" (1980, p. 22), paralleling my hypothesis regarding HR deceleration in the OR context. Barry (1983) found evidence that primary bradycardia coexisted with HR deceleration in an OR study, and that both phenomena were similarly independent of stimulus intensity and novelty. This suggested that the cardiac 
response in the OR context, which at least begins as a primary bradycardia, is either nothing more than primary bradycardia or involves some additional, but as yet unknown, process.

The proposition that HR deceleration in the OR context is solely a primary bradycardia was examined in a recent report from this laboratory (Barry, 1984a). The independence of stimulus and situational determinants discussed by Bohlin and Kjellberg (1979) and Velden and Schumacher (1979) was also explored. Situational requirements were imposed to produce a two-component cardiac response: In Bohlin and Kjellberg's terms, a single stimulus was expected to elicit D1 (reflecting stimulus registration) preceding, and partly obscured by, A (reflecting processing requirements). These hypothetical processes were modeled by orthogonal trend components over beats. Of major interest were the findings linking D1 with primary bradycardia. D1 was independent of stimulus durations from $0.1 \mathrm{sec}$ (used by the Laceys in their work on primary bradycardia) to $2.1 \mathrm{sec}$ (similar to durations commonly used in our OR work). Also, over the first two beats, D1 showed the interaction between beat and cardiac phase at stimulus onset that has been established as characteristic of primary bradycardia. Finally, after those two beats, D1 showed only a return towards baseline, ruling out the existence of another source of HR deceleration apart from that of primary bradycardia. These results support the identification of cardiac deceleration in the OR context as a primary bradycardia.

That study presented each subject with more than 192 stimuli. Because stimulus onset was randomly related to cardiac phase, such a large number was necessary to allow adequate numbers of the various stimulus types to fall in each quartile of the cardiac cycle. Data were then collapsed within subject to obtain mean response profiles for each stimulus type presented at each quartile of the cardiac cycle. This precluded an analysis of trials effects in $A$ and D1. The presence or absence of such effects in $\mathrm{D} 1$, as discussed above, is of some contention in the literature. Also, if A reflects processing load, the development of this processing load over trials should be apparent as an increase in A with stimulus repetition. The present report examines these trials effects in a subset of the data from Barry (1984a).

\section{METHOD}

\section{Subjects}

Twenty university students ( 8 males, 12 females), between the ages of 20 and 25 years, participated as one means of satisfying a course requirement in educational psychology at the University of New South Wales.

\footnotetext{
Apparatus and Procedure

Stimuli consisted of $2400-\mathrm{Hz}$ tones, in random combinations of 33 or $50 \mathrm{~dB}$ (re $20 \mu \mathrm{N} / \mathrm{m}^{2}$ ) with $0.1-, 1.1-$, or 2.1 -sec duration, presented via circumaural headphones. Interstimulus intervals varied randomly among 9,11 , and $13 \mathrm{sec}$. There were four blocks of stimuli, each containing between 48 and 52 stimuli on a random basis; these were separated by 2 -min rest periods. The subjects were
}

asked to count the number of stimuli in each block and were promised 50 cents for each correct block total. The experimenter entered the subject cubicle in each rest period to check this total and pay the promised reward if correct. Only subjects with at least three correct block totals were included in the study.

The EKG from standard lead 1 triggered an R-wave peak detector, the output from which was used to compute $R-R$ intervals for one prestimulus and five poststimulus beats (the first of which contained the stimulus onset) for each stimulus presentation. These were recorded on floppy disk for off-line analysis. The procedure is described in more detail in Barry (1984a). For the purposes of this report, the first eight presentations of each stimulus type were analyzed.

\section{RESULTS}

The mean response profile over beats, expressed in terms of deviations from the prestimulus period, is shown in Figure 1. Inspection suggests that the cardiac response obtained here, as expected from previous work, consisted of a small deceleratory HR response (D1) partly obscured by a larger acceleratory response (A). Hypothetical versions of these component responses, which add to form the observed response, are also included in Figure 1. Following Barry (1984a), these components were modeled by orthogonal trend components over beats. A was modeled as a linear trend, and D1 as the sum of quadratic and cubic trends. The models, and the effects upon them of the independent variables, were examined in a four-way (over beats, intensity, duration, and trials) repeated-

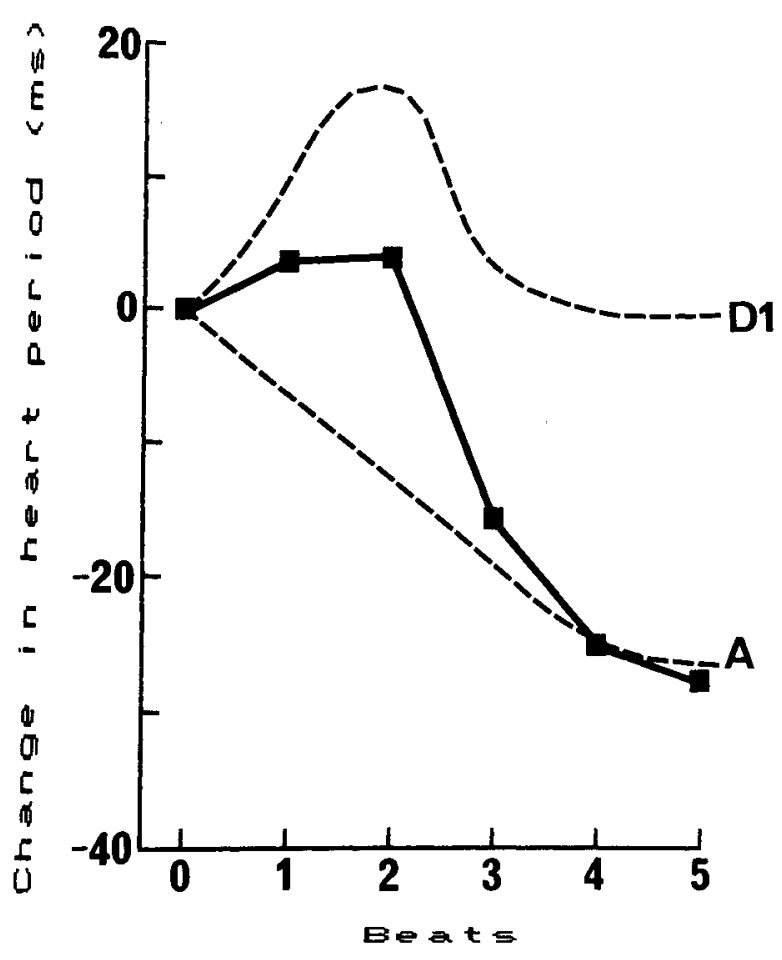

Figure 1. The points connected by heavy lines indicate mean changes in heart period from the prestimulus beat (labeled 0 ) for each of the five poststimulus beats. The broken lines represent models of $\mathrm{D1}$ and $\mathrm{A}$ that add to produce the observed response. 
measures planned contrasts ANOVA carried out on the 288 data points from each subject. Within beats, the linear trend was significant ${ }^{1}[\mathrm{~F}(1,19)=25.88$, MSe $=$ $31,908.15]$, as were the quadratic and cubic trends $[\mathrm{F} 1,19)$ $=4.94$, MSe $=11,207.15$, and $F(1,19)=10.98$, MSe $=9,652.83$, respectively]. Together, these three trends accounted for $97 \%$ of the systematic variance within beats.

From the four-way ANOVA, the effects of stimulus duration, intensity, and novelty upon D1 and A may be found in the interactions between the independent variables and the three trend components over beats. There were no significant effects of stimulus duration, either upon D1 $[\mathrm{F}(2,38)=0.32$ and 2.00 , MSe $=3,548.70$ and $3,128.08$, for the quadratic and cubic trend components, respectively] or upon $\mathrm{A}[\mathrm{F}(2,38)=1.39$, MSe $=$ $4,722.17]$. Similarly, stimulus intensity effects failed to approach significance for both $\mathrm{D} 1[\mathrm{~F}(1,19)=0.21$ and 2.29 , MSe $=3,775.18$ and 1,973.66, for the quadratic and cubic trends, respectively $]$ and $\mathrm{A}[\mathrm{F}(1,19=1.75$, MSe $=4,094.41]$. Trials effects were examined in terms of linear and quadratic trends over trials. For the quadratic component of $\mathrm{D} 1$, both trends over trials failed to approach significance $[F(1,19)=2.23$ and 0.69, MSe $=$ $4,773.09$ and 3,751.61, for the linear and quadratic trends over trials, respectively]. This was also true for the cubic component of D1, with both Fs less than unity. This relative insensitivity of D1 to reductions in novelty is apparent in the top part of Figure 2. In contrast, A was found

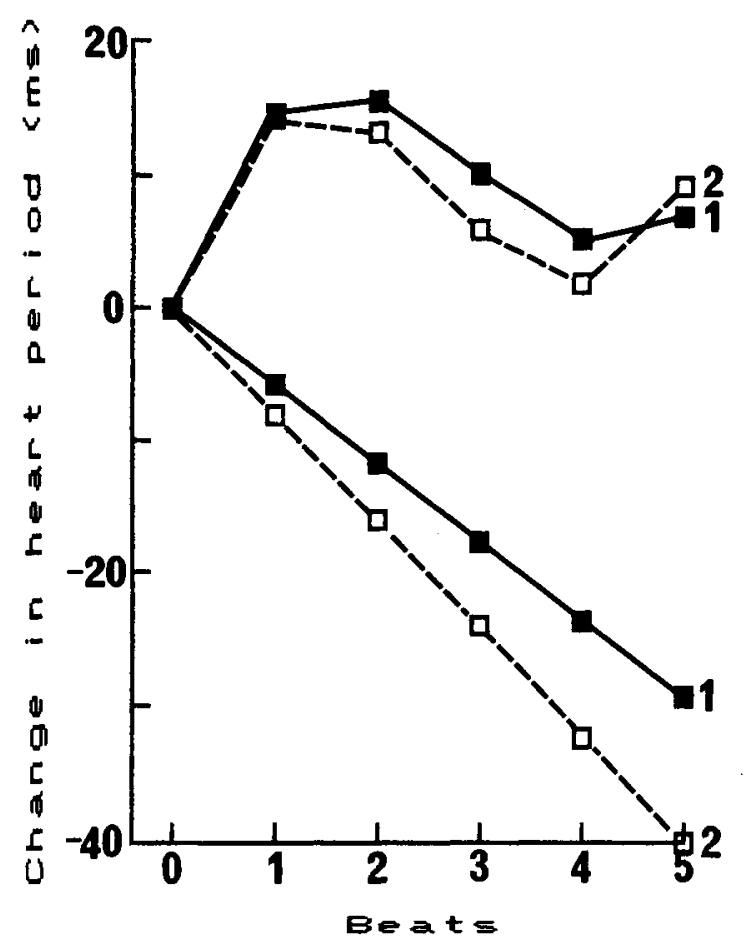

Figure 2. Models of D1 (top two curves) and A (bottom two curves), derived from the changes in period over beats. Numbers 1 and 2 refer to the mean responses in the first and second halves of the study, respectively.
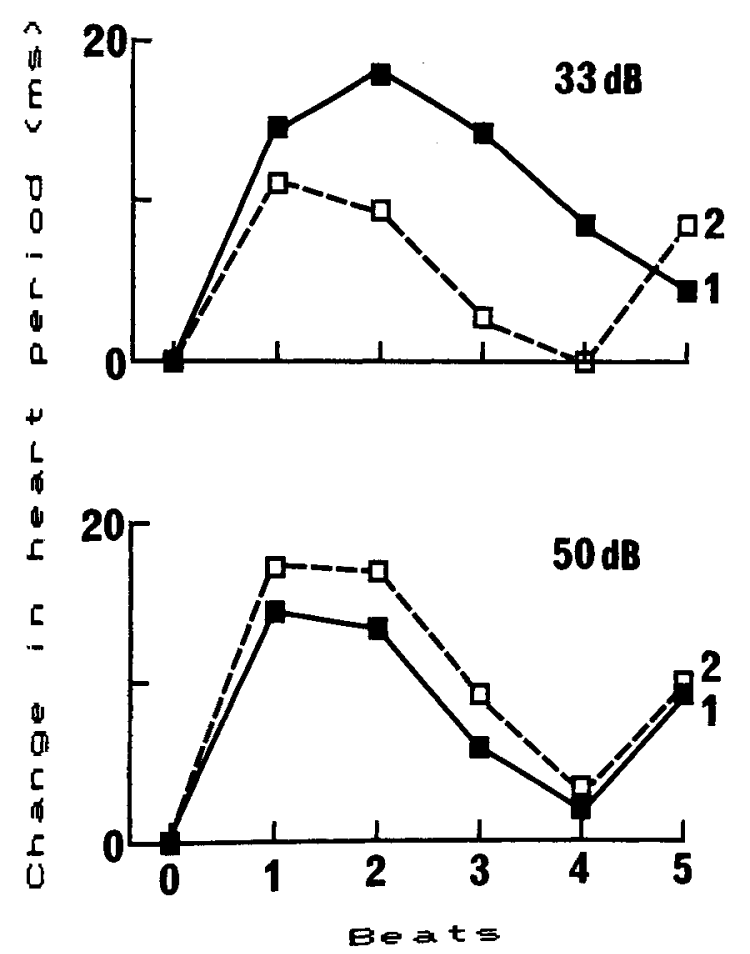

Figure 3. Models of D1 over beats for each stimulus intensity (33 and $50 \mathrm{~dB}$ ) for each half of the study (indicated by the numbers 1 and 2).

to exhibit a significant linear trials effect $[F(1,19)=7.76$, $\mathrm{MSe}=5,794.82]$ without a significant quadratic trials effect $[F(1,19)=1.80, \mathrm{MSe}=4,997.61]$. The lower part of Figure 2 indicates that A increased over trials.

D1 was also significantly affected by an interaction between trials and intensity. This was apparent in the quadratic trend component over beats as an interaction between the intensity effect and the linear trend over trials $[F(1,19)$ $=5.63, \mathrm{MSe}=5,251.22]$. Also, in the cubic trend component over beats, the interaction between intensity and the quadratic trend over trials was significant $[F(1,19)=$ $9.23, \mathrm{MSe}=1,505.82]$. Figure 3 indicates that this interaction may be attributed to an increase over trials in D1 elicited by 50-dB stimuli, whereas D1 elicited by 33-dB stimuli decreased over trials. This interaction failed to approach significance for $A$, and there were no other significant interactions affecting D1 or A.

\section{DISCUSSION}

The data reported here were derived from portions of the data set analyzed in Barry (1984a), and consisted of cardiac responses to the first eight presentations of each of six stimulus types. These stimulus types varied intensity within the OR domain ( 33 and $50 \mathrm{~dB}$ ) and stimulus duration from 0.1 to $2.1 \mathrm{sec}$. The present findings, indicating the absence of significant main effects of stimulus intensity and duration, in both D1 and A, confirm the results reported in Barry (1984a). 
The major focus of this study was the effect of trials upon the cardiac response components. No significant effect of stimulus repetition was obtained with D1, but A was found to increase significantly over trials. Both these findings support the conceptualization of the phasic cardiac response developed in this laboratory. Thus, D1, which is relatively insensitive to variation in stimulus parameters, appears to reflect registration of the occurrence of the stimulus, the initial stage in the stimulus processing that precedes OR elicitation. Previous work (Barry, 1984a) has supported the identification of D1 with the Laceys' primary bradycardia, strengthening this interpretation. The present finding that A increased over trials supports the hypothesis that this component reflects processing demand, but further exploration of its determinants is necessary to confirm this hypothesis.

These differential trials effects in the two response components are of some importance to the conceptualization of the evoked cardiac response as an OR indicator. Clearly, cardiac deceleration, as reflected in D1, cannot stand as an OR indicator because of its failure to reflect stimulus intensity and novelty. However, the composite cardiac response $(\mathrm{A}+\mathrm{D} 1)$ may appear to decrease in size over trials because of the increase in A. Such a result was reported in Barry (1983), based on an ECR elicited by low-level stimuli that had to be attended. HR deceleration was the major feature of the ECR, with some HR acceleration apparent in later beats. Over the first 5 poststimulus beats, there were no significant trials effects, but over the next 5 beats, the linear trend component increased. It thus appears necessary to separate trend components of the ECR in any discussion of trials effects that are of theoretical importance. Failure to do so in the past may have contributed to some claims supporting the identification of D1 as an OR indicator.

The interaction between intensity and trials, shown in Figure 3, should be mentioned briefly. Although D1 elicited by the 33-dB stimuli decreased over trials, this cannot be taken as support for the ECR-as-OR position without some adequate explanation for the corresponding increase in the D1 elicited by the 50 -dB stimuli. Since a 50-dB stimulus is well within the intensity limits usually associated with the OR/DR transition, a DR-based explanation does not appear feasible. No theoretical basis for this interaction is immediately apparent.

\section{REFERENCES}

BARRY, R. J. (1977a). The effect of "significance" upon indices of Sokolov's orienting response: A new conceptualization to replace the OR. Physiological Psychology, 5, 209-214.
BARRY, R. J. (1977b). Failure to find evidence of the unitary OR concept with indifferent low-intensity stimuli. Physiological Psychology, $5,89-96$

BARRY, R. J. (1981). Signal value and preliminary processes in OR elicitation. Pavlovian Journal of Biological Science, 16, 144-150.

BARRY, R. J. (1982). Novelty and significance effects in the fractionation of phasic OR measures. A synthesis with traditional OR theory. Psychophysiology, 19, 28-35.

Barry, R. J. (1983). Primary bradycardia and the evoked cardiac response in the OR context. Physiological Psychology, 11, 135-140.

BARRY, R. J. (1984a). The evoked cardiac response under processing load. Physiological Psychology, 12, 35-40.

BARry, R. J. (1984b). Preliminary processes in OR elicitation. Acta Psychologica, 55, 109-142.

BoHLIN, G., \& KJELlBERG, A. (1979). Orienting activity in two-stimulus paradigms as reflected in heart rate. In H. D. Kimmel, E. H. van Olst, \& J. F. Orlebeke (Eds.), The orienting reflex in humans. Hillsdale, $\mathrm{NJ}$ : Erlbaum.

Coles, M. G. H., \& DunCan-Johnson, C. C. (1975). Cardiac activity and information processing: The effects of stimulus significance and detection and response requirements. Journal of Experimental Psychology: Human Perception and Performance, 1, 418-428.

Graham, F. K., \& Clifton, R. K. (1966). Heart-rate change as a component of the orienting response. Psychological Bulletin, 65, 305-320.

HuLSTIJN. W. (1978). Habituation of the orienting response as a function of arousal induced by three different tasks. Biological Psychol ogy, 7, 109-124.

LACEY, B. C., \& LACEY, J. I. (1977). Change in heart period: A function of sensorimotor event timing within the cardiac cycle. Physiological Psychology, 5, 383-393

LACEY, B. C., \& LACEY, J. I. (1978). Two-way communication between the heart and the brain: Significance of time within the cardiac cycle. American Psychologist, 33, 99-113.

LACEY, B. C., \& LACEY, J. I. (1980). Cognitive modulation of timedependent primary bradycardia. Psychophysiology, 17, 209-221.

LACEY, J. I. (1967). Somatic response patterning and stress: Some revisions of activation theory. In M. H. Appley \& R. Trumbull (Eds.), Psychological stress: Issues in research. New York: Appleton-CenturyCrofts.

von Olst, E. H., Heemstra, M. L., \& Ten Kortenaar, T. (1979). Stimulus significance and the orienting reaction. In H. D. Kimmel, E. H. van Olst, \& J. F. Orlebeke (Eds.), The orienting reflex in humans. Hillsdale, NJ: Erlbaum.

Velden, M., \& Schumacher, R. (1979). Orienting and defensive cardiac responses. In H. D. Kimmel, E. H. van Olst, \& J. F. Orlebeke (Eds.), The orienting reflex in humans. Hillsdale, NJ: Erlbaum.

Walter, G. F., \& Porges, S. W. (1976). Heart rate and respiratory responses as a function of task difficulty: The use of discriminant anal ysis in the selection of psychologically sensitive physiologica responses. Psychophysiology, 13, 563-571.

\section{NOTE}

1. Significance level used is $p<.05$.

(Manuscript received May 16, 1984; accepted for publication October 1, 1984.) 